\title{
ESTUDO DE FILAS EM CAIXAS RÁPIDOS DE UM SUPERMERCADO UTILIZANDO SIMULAÇÃO COMPUTACIONAL
}

\author{
Ana Cláudia Souza ${ }^{1}$ \\ Lorena Aparecida Tosoni ${ }^{2}$ \\ Rafaela Cristina Canassa ${ }^{3}$ \\ Márcia de Fátima Morais ${ }^{4}$
}

\begin{abstract}
RESUMO: O presente trabalho trata do estudo das filas em caixas rápido de um Supermercado por meio da simulação computacional. O estudo teve por objetivo verificar o comportamento das filas nos caixas rápidos do Supermercado X localizado na cidade de Campo Mourão - PR. Esta pesquisa classifica-se, quanto aos fins, como descritiva e explicativa e, quanto aos meios como bibliográfica, de campo e estudo de caso. A coleta de dados foi realizada em horários de pico, devido ao congestionamento dos caixas rápidos nesse horário. Com a pesquisa, foi possível verificar que, ao operar com um número maior de caixas, ocorrerá uma mudança significativa em relação ao número de clientes na fila, ao tempo médio de clientes na fila e no sistema. Sugere-se o reposicionamento dos caixas, para formação de filas individuais, bem como também alocação de colaboradores extra, para empacotamento de mercadorias, para melhor atendimento dos clientes.
\end{abstract}

Palavras-Chave: Pesquisa Operacional, Filas, Simulação Computacional, Supermercado.

\begin{abstract}
This paper deals with the study of the queues rapidly boxes of a Supermarket by computer simulation. The study aimed to verify the behavior of the rows in boxes of fast X Supermarket located in Campo Mourão - PR. This research is classified, as to the purposes, such as descriptive and explanatory, and as to the means as literature, field and case study. Data collection was carried out during peak hours, due to congestion of rapid boxes at this time. Through research, we found that when operating with a larger number of cases, there will be a significant change in the number of customers in the queue, the average time customers in the queue and the system. It is suggested repositioning of boxes, to form individual lines and allocating extra staff for packing goods, for better customer service.
\end{abstract}

Keywords: Operational Research, Queues, Computer Simulation, Supermarket.

\footnotetext{
${ }^{1}$ Universidade Estadual do Paraná - UNESPAR/

${ }^{2}$ Universidade Estadual do Paraná - UNESPAR/FECILCAM lorena.tosoni@hotmail.com.

${ }^{3}$ Universidade Estadual do Paraná - UNESPAR/

${ }^{4}$ Universidade Estadual do Paraná - UNESPAR/
}

ReLAInEP - Revista Latino-America de Inovação e Engenharia de Produção, Curitiba, PR, Brasil, v. 3, n. 4, p. 203-221, 2015. 
RESUMEN: Este artículo se ocupa del estudio de las colas rápidamente cajas de un supermercado por simulación por ordenador. El estudio tuvo como objetivo verificar el comportamiento de las filas en cajas de rápido X Supermercado ubicado en Campo Mourao PR. Esta investigación se clasifica, en cuanto a los fines, como descriptivo y explicativo, y en cuanto a los medios como la literatura, el campo y el estudio de caso. La recolección de datos se llevó a cabo de las horas pico, debido a la congestión de las cajas rápidas en este momento. A través de la investigación, se encontró que cuando se opera con un mayor número de casos, habrá un cambio significativo en el número de clientes en la cola, los clientes de tiempo promedio en la cola y el sistema. Se sugiere reposicionamiento de cajas, para formar líneas individuales y también la asignación de personal adicional para el embalaje de mercancías, para un mejor servicio al cliente.

Palabras-Clave: Investigación Operativa, Colas, Simulación Computacional, Supermercado.

\section{INTRODUÇÃO}

Dentre as diversas Áreas de conhecimento da Engenharia de Produção, encontra-se a Pesquisa Operacional (PO). Segundo a ABEPRO (2008) a PO é a área da Engenharia de Produção que trata da resolução de problemas reais envolvendo situações de tomada de decisão, através de modelos matemáticos habitualmente processados computacionalmente (ABEPRO, 2008).

A PO é uma abordagem científica para tomada de decisões, seu objetivo é determinar como melhor projetar e operar um sistema, utilizando modelos matemáticos (ARENALES et al., 2011). Uma das subáreas da PO de acordo com a ABEPRO (2008) é a Modelagem, Simulação e Otimização, foco deste trabalho. A Simulação, que permite transcrever o funcionamento de um sistema real, admitindo montar um modelo que melhor represente o sistema em estudo (PRADO, 1999).

A simulação pode ser utilizada em diferentes locais e nas áreas mais diversificadas no mundo atual, tanto na produção, quanto na manufatura. Dentre os setores de aplicações da simulação, Prado (1999) destaca supermercados, bancos e escritórios. A simulação em supermercados pode ser empregada para "dimensionar o número de caixas de modo que as filas se mantenham abaixo de um valor especificado" como também, "avaliar o uso de caixas especiais tais como caixas rápidos" (PRADO, 1999, p. 19). O estudo de filas, segundo Taha (2008) visa quantificar o fenômeno da espera em filas usando medidas representativas de desempenho como o tamanho médio de uma fila, o tempo médio de espera em fila e a taxa média de utilização das instalações.

ReLAInEP - Revista Latino-America de Inovação e Engenharia de Produção, Curitiba, PR, Brasil, v. 3, n. 4, p. 203-221, 2015. 
Diante do exposto, o trabalho aqui apresentado teve como objetivo realizar um estudo do comportamento da fila dos caixas rápidos do Supermercado X, situado na cidade de Campo Mourão/PR, por meio da Simulação Computacional. A pesquisa caracteriza-se quanto aos fins, como descritiva e explicativa e, quanto aos meios, como bibliográfica, de campo e estudo de caso. Para a realização deste trabalho, utilizou-se o método de abordagem qualitativo e quantitativo. Para a modelagem e simulação do sistema de filas dos caixas rápidos do Supermercado X, cujos propósitos foram apenas acadêmicos, foi utilizada a versão Training/Evaluation Mode do Software Arena 14.0 da Rockwell Software.

O presente artigo encontra-se estruturado em cinco seções. A primeira seção, a introdução, contextualização do problema investigado e expõem o objetivo do estudo. Logo em seguida encontra-se o referencial teórico, que apresenta conceitos acerca de sistemas de filas e simulação computacional. Na terceira seção apresenta-se a revisão de literatura. Na quarta seção, o estudo de filas nos caixas rápidos do Supermercado X é apresentado. Por fim, na quinta seção apresentam-se as considerações finais, seguida das referências bibliográficas utilizadas.

\section{REFERENCIAL TEÓRICO}

\subsection{Sistemas de Filas}

Um sistema de filas é um processo de nascimento-morte com uma população composta de usuários esperando para serem atendidos e sendo atendidos, entretanto um nascimento ocorre quando um usuário chega no estabelecimento de prestação de serviços, uma morte ocorre quando um usuário deixa este estabelecimento (BRONSON, 1985; TAHA, 2008).

Aguardar em filas é algo comum, tanto para realizar compras no supermercado, pagar uma conta no banco, esperar uma consulta nos hospitais, esperar para entrar no cinema ou teatro, entre outros. Filas são situações inevitáveis, e estão em nosso cotidiano e em uma classe muito grande de sistemas (MOREIRA, 2007; ELLENRIEDER, 1971).

Entretanto não apenas pessoas esperam em filas, em indústrias este fato ocorre com frequência, peças e produtos podem estar aguardando processamento. Como também navios podem estar esperando para entrar nos portos e aviões podem estar aguardando autorização para aterrissar (MOREIRA, 2007).

ReLAInEP - Revista Latino-America de Inovação e Engenharia de Produção, Curitiba, PR, Brasil, v. 3, n. 4, p. 203-221, 2015. 
Estudos de Filas tratam de problemas de congestionamento de sistemas, onde a característica principal é a presença do cliente em busca de serviços. De forma geral um sistema de filas é composto por elementos que querem ser atendidos e por um posto de serviço que eventualmente deve esperar até que este posto esteja livre (ANDRADE, 2000).

Conforme Pereira Júnior (2010), as filas apresentam as seguintes características: Clientes e tamanho da população; Processo de chegada representado pelo intervalo de chegada (IC) e ritmo de chegada $(\lambda)$; Processo de atendimento; Capacidade dos recursos, sejam máquinas, atendentes, entre outros; Disciplina da fila; Número de filas; Tamanho médio da fila, ou seja, o número médio de clientes em fila (NF); Tamanho máximo da fila; Tempo médio de espera na fila: $\sum \mathrm{TF}\left(\right.$ Tempo em fila)/ $\mathrm{N}^{\circ}$ clientes atendidos ( PEREIRA JÚNIOR, 2010; MORAIS, 2013).

Existem vários fatores que limitam a operação de um sistema, isto é, sua interferência é tanta que o desempenho do sistema passa a ser função deles (ANDRADE, 2002). Esses fatores são classificados em quatro itens: forma de atendimento, modo de chegada, disciplina da fila e estrutura do sistema.

Geralmente, os postos de atendimento são constituídos por pessoas, instalações e equipamentos que necessitam atuar em sintonia, de modo a proporcionar um bom serviço ao cliente. Dessa maneira, existem diversos meios que aprimoram o desempenho do sistema, tais como: dimensionamento da capacidade, treinamento de atendentes, rotinas administrativas, sistemas de informações, entre outros (ANDRADE, 1989).

A forma de chegadas de clientes em um sistema ocorre, na maioria das vezes de modo aleatório, isto é, o número de clientes que chegam por unidade de tempo varia conforme o comportamento do fluxo de chegadas. Para isso, é importante fazer um levantamento estatístico com objetivo de verificar o processo de chegadas dos clientes (ANDRADE, 1989; MORAIS, 2013).

A disciplina da fila é constituída por regras que caracterizam a sequência em que os clientes serão atendidos, esse atendimento pode ocorrer "pela ordem de chegadas (o primeiro que chegar é o primeiro a ser atendido), pela ordem inversa de chegada (o último a chegar é o primeiro a ser atendido), pelo atendimento com prioridade para certas classes" (ANDRADE, 1989, p. 193; MORAIS, 2013).

ReLAInEP - Revista Latino-America de Inovação e Engenharia de Produção, Curitiba, PR, Brasil, v. 3, n. 4, p. 203-221, 2015. 


\section{ReLAlnEP}

Os sistemas de filas possuem estruturas variadas, e cada caso demanda um estudo analítico diferente. As estruturas podem classificadas como: um sistema de uma fila e um canal, um sistema de uma fila e diversos canais e um sistema complexo de filas e canais em série e em paralelo (ANDRADE, 1998). A Figura 1 ilustra um sistema de uma fila e três canais.

Figura 1- Sistema de uma fila e três canais.

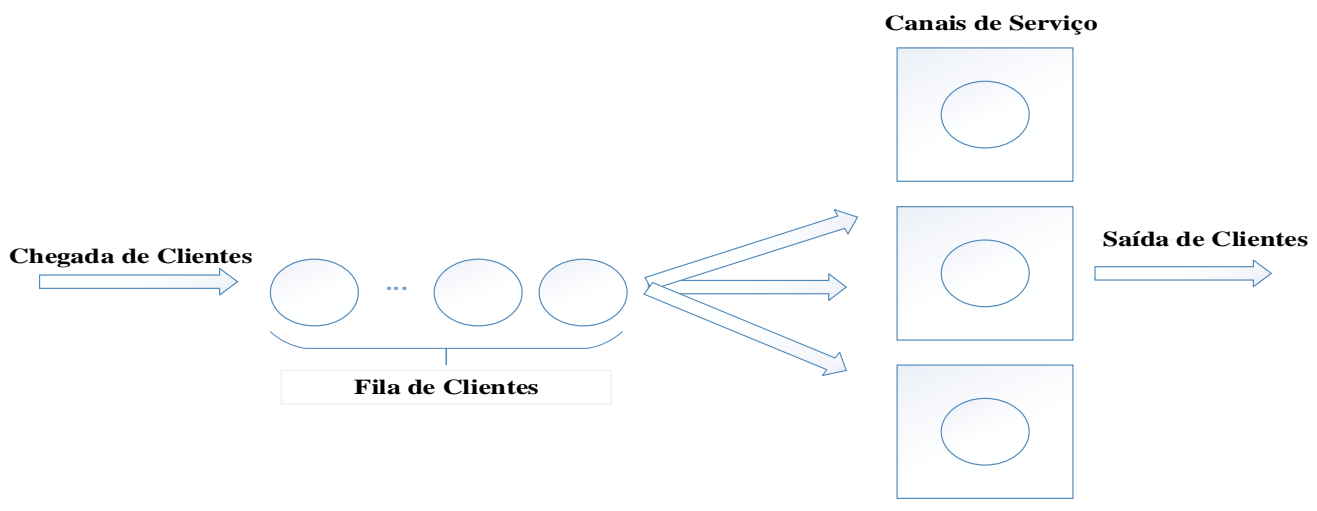

Fonte: Andrade (2002).

\subsection{Simulação Computacional}

Miyagi (2006, p. 2) define a simulação como sendo a imitação de sistema do mundo real que envolve a criação de uma "história artificial de um sistema para a análise de suas características operacionais".

Conforme Assis (2010, p. 2) a simulação imita "a realidade sem correr os riscos, os custos e o tempo que resultariam se tivéssemos de experimentar". Para isso é necessário criar "um modelo matemático que descreva o comportamento ao longo do tempo do sistema que vamos estudar". De acordo com Brighenti (2006) o processo de simulação segue um método científico, onde são apresentadas etapas a serem seguidas, como podemos observar na Figura 2.

ReLAInEP - Revista Latino-America de Inovação e Engenharia de Produção, Curitiba, PR, Brasil, v. 3, n. 4, p. 203-221, 2015. 


\section{ReLAInEF}

Figura 2 - Método científico aplicado a simulação.

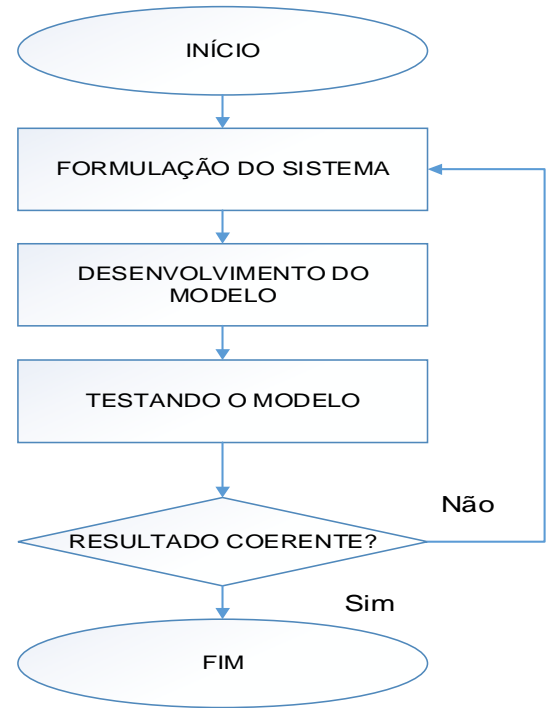

Fonte: Harrell (2000 apud Brighenti, 2006).

A simulação possui a capacidade de fornecer resultados para as análises mais elaboradas a respeito do sistema de modo que a interpretação desse sistema seja mais profunda e abrangente (BRIGHENTI, 2006).

$\mathrm{Na}$ área de modelagem e simulação, de acordo Sampaio e Oliveira (2013) os softwares mais conhecidos e utilizados são: Automod, Micro Saint, Witness, Arena, SimPy e Promodel. Especificamente para este trabalho foi utilizado Software Arena versão 14.0.

\subsubsection{O software Arena}

O Software Arena é uma ferramenta bastante empregada na simulação de processos. $\mathrm{O}$ Arena possui diversas ferramentas e funcionalidades que são utilizadas para descrever uma aplicação real, por meio dele é possível ter uma maior visualização do "sistema a ser modelado como constituído de um conjunto de estações de trabalho que prestam serviços a clientes, que se movem através do sistema" (PRADO, 1999, p. 26).

De acordo com Prado (1999) e Silveira, Silva e Berlamino (2006), o Software Arena, possui ferramentas muito úteis, tais como: Analisador de dados de entrada (Input Analyser), Analisador de resultados (Output Analyser), Visualizador de Simulação (Arena Viewer) e Execução de Lotes (Scenario Manager). Dentre os citados, no estudo de aqui apresentado,

ReLAInEP - Revista Latino-America de Inovação e Engenharia de Produção, Curitiba, PR, Brasil, v. 3, n. 4, p. 202-220, 2015. 


\section{ReLAInEP}

faz-se apenas uso do Input Analyser.

O Input Analyser "permite analisar dados reais do funcionamento do processo e escolher a melhor distribuição estatística que se aplica a eles e essa distribuição pode ser incorporada diretamente ao modelo" e o Output Analyzer "é uma ferramenta com diversos recursos que permite analisar dados coletados durante a simulação, sendo que esta análise pode ser gráfica, e tem ainda recursos para efetuar importantes comparações estatísticas" (PRADO, 1999, p.26).

A linguagem de simulação incorporada no Software Arena é a Siman V, onde os sistemas a serem modelados podem pertencer a tais áreas: manufatura, serviços, sistemas de transportes, logística, computadores, etc. (MORAIS, 2013). A Figura 3 mostra a tela inicial do Software Arena.

Figura 3 - Tela Inicial do Software

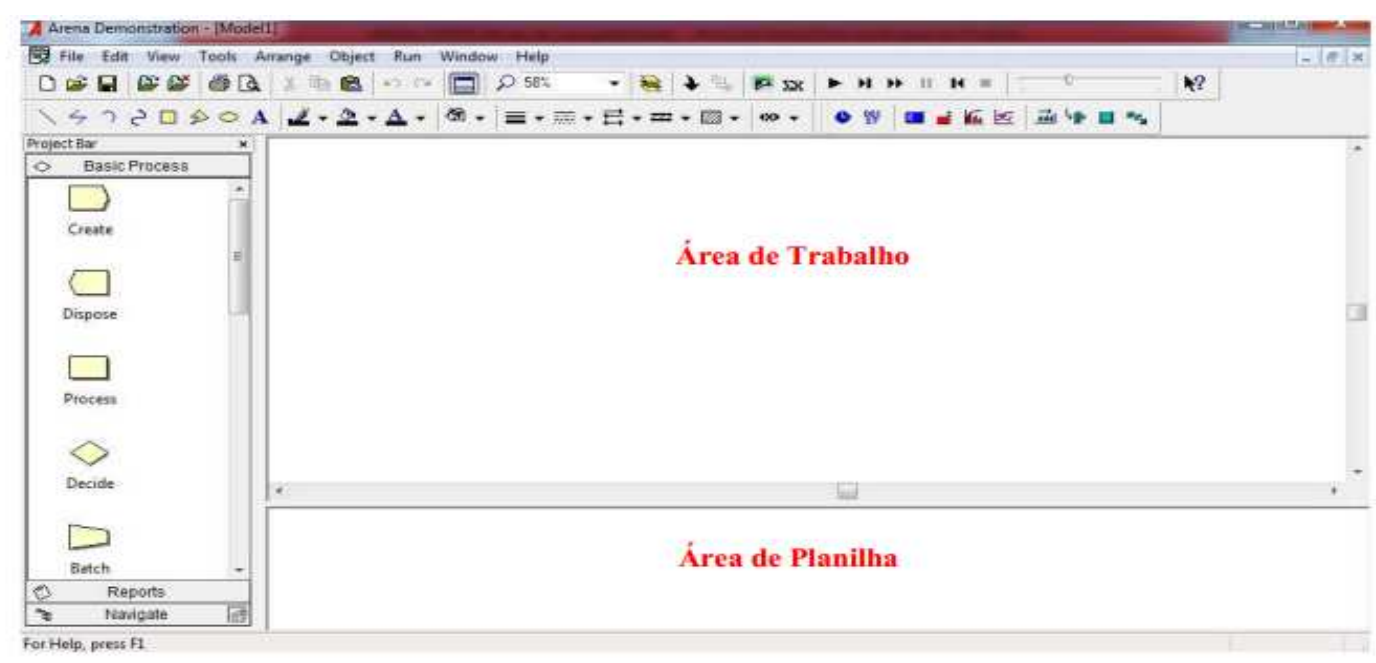

Arena versão 14.0.

O diferencial do Software Arena é a tecnologia utilizada, ou seja, os templates, definidos como "uma coleção de objetos/ferramentas de modelagem, que permitem ao usuário, descrever o comportamento do processo em análise, através de respostas às perguntas préelaboradas de maneira visual e interativa" (PARAGON, 2006 apud SILVEIRA, SILVA e BELARMINO, 2006, p. 33).

Cada template é composto por vários blocos e cada bloco tem uma função. Os blocos do

ReLAInEP - Revista Latino-America de Inovação e Engenharia de Produção, Curitiba, PR, Brasil, v. 3, n. 4, p. 202-220, 2015. 


\section{ReLAlnEF}

template basic process são os seguintes: create, dispose, process, decide, batch e assign. (SILVEIRA, SILVA e BELARMINO, 2006). Esses blocos podem ser visto na Figura 3 acima. Os blocos utilizados nesse trabalho foram create, process e dispose.

\section{REVISÃO DE LITERATURA}

$\mathrm{Na}$ literatura especializada foram pesquisados trabalhos publicados nos últimos 6 anos, ou seja, no período compreendido entre os anos de 2007 a 2014. Estudos de filas orientados a Supermercados foram verificados em Rinaldi (2007), Alencar et al. (2010), Schneiders, Desconzi e Ruppenthal (2012) e Scarp, Torres e Silva (2013). O Quadro 1 explicita os objetivos destes trabalhos.

Quadro 1 - Trabalhos orientados ao estudo de filas em supermercados

\begin{tabular}{|l|l|}
\hline Referência & Objetivo \\
\hline Rinaldi (2007) & $\begin{array}{l}\text { Mostrar que o tempo de espera na fila de caixas de supermercados } \\
\text { assume grande importância na rapidez de atendimento e, portanto, } \\
\text { para o nível de serviço dos clientes. }\end{array}$ \\
\hline Alencar et al (2010) & $\begin{array}{l}\text { Problemática das filas nos caixas rápidos de um supermercado, por } \\
\text { intermédio da modelagem matemática fundamentada em teoria das } \\
\text { filas. }\end{array}$ \\
\hline $\begin{array}{l}\text { Schneiders, Desconzi } \\
\text { e Ruppenthal (2012) }\end{array}$ & $\begin{array}{l}\text { Verificar o número de clientes à espera por atendimento e a taxa de } \\
\text { ociosidade de um supermercado no município de Doutor Maurício } \\
\text { Cardoso/RS }\end{array}$ \\
\hline $\begin{array}{l}\text { Scarp, Torres e Silva } \\
(2013)\end{array}$ & $\begin{array}{l}\text { Representar o sistema dos caixas de atendimento de um supermerca- } \\
\text { do da cidade de Viçosa, MG, por meio da simulação, comparando os } \\
\text { resultados obtidos pela simulação com a Teoria das Filas. }\end{array}$ \\
\hline
\end{tabular}

Fonte: Elaborado pelos autores

\section{ESTUDO DE FILAS NOS CAIXAS RÁPIDOS DO SUPERMERCADO}

\subsection{O Supermercado X}

Fundado em 25 de março de 1964 por dois irmãos, sendo assim caracterizada por ser uma empresa familiar, o Supermercado $\mathrm{X}$ vem crescendo nos últimos anos e ganhando credibilidade e fidelidade dos seus consumidores, que são característicos das classes média e baixa.

O Supermercado $X$ conta com 52 funcionários, divididos em duas equipes que trabalham de Segunda-Feira a Sábado. A primeira equipe trabalha das $8 \mathrm{~h} 00 \mathrm{~min}$ às $18 \mathrm{~h} 00 \mathrm{~min}$, e a segunda, trabalho das 9h30min às $19 \mathrm{~h} 30 \mathrm{~min}$.

ReLAInEP - Revista Latino-America de Inovação e Engenharia de Produção, Curitiba, PR, Brasil, v. 3, n. 4, p. 202-220, 2015. 
Os caixas são separados em caixas normais e caixas rápidos, distribuídos respectivamente em 9 e 4 caixas. Geralmente, a empresa opera apenas com 2 caixas rápidos, atendendo exclusivamente consumidores com até 10 itens, sendo o operador do caixa responsável por embalar as mercadorias.

\subsection{Coleta e Tratamento dos Dados}

Verificando que o horário de maior afluência de clientes ao Supermercado X concentrase entre $17 \mathrm{~h} 30 \mathrm{~min}$ e $18 \mathrm{~h} 30 \mathrm{~min}$, os tempos para análise do sistema de filas dos caixas rápidos foram coletados neste intervalo de tempo, nos dias 23/10/2013 e 30/10/2013. Foram coletadas 150 amostras para os tempos entre chegadas de clientes na fila dos caixas rápidos (TABELA 1) e 150 amostras para os tempos de atendimento dos clientes nos caixas rápidos (TABELA 2).

Tabela 1 - Tempos entre chegadas de clientes (segundos) na fila dos caixas rápidos.

\begin{tabular}{|l|l|l|l|l|l|l|l|l|l|}
\hline 46 & 50 & 6 & 21 & 13 & 18 & 68 & 27 & 119 & 24 \\
\hline 60 & 39 & 72 & 54 & 0 & 5 & 2 & 14 & 2 & 206 \\
\hline 16 & 41 & 34 & 93 & 170 & 13 & 1 & 30 & 201 & 71 \\
\hline 41 & 9 & 36 & 102 & 45 & 4 & 56 & 3 & 43 & 8 \\
\hline 10 & 59 & 67 & 59 & 24 & 16 & 33 & 13 & 48 & 56 \\
\hline 20 & 3 & 1 & 28 & 39 & 33 & 41 & 89 & 5 & 7 \\
\hline 8 & 58 & 0 & 27 & 13 & 66 & 78 & 4 & 131 & 41 \\
\hline 12 & 13 & 55 & 2 & 78 & 58 & 14 & 18 & 31 & 7 \\
\hline 72 & 29 & 69 & 77 & 52 & 24 & 48 & 98 & 32 & 100 \\
\hline 4 & 1 & 17 & 61 & 24 & 3 & 8 & 159 & 57 & 21 \\
\hline 34 & 55 & 41 & 120 & 68 & 115 & 28 & 28 & 26 & 33 \\
\hline 0 & 10 & 1 & 1 & 115 & 61 & 13 & 48 & 9 & 25 \\
\hline 138 & 26 & 85 & 118 & 25 & 44 & 1 & 12 & 16 & 20 \\
\hline 143 & 54 & 13 & 71 & 74 & 126 & 70 & 4 & 25 & 19 \\
\hline 132 & 22 & 14 & 16 & 36 & 10 & 42 & 5 & 8 & 134 \\
\hline
\end{tabular}

Assumindo que os tempos de atendimento seguem uma Distribuição Exponencial e que os tempos de atendimento seguem uma Distribuição Normal, foi utilizada a ferramenta Input Analyzer do Software Arena, para identificar os parâmetros associados a distribuição de probabilidade.

ReLAInEP - Revista Latino-America de Inovação e Engenharia de Produção, Curitiba, PR, Brasil, v. 3, n. 4, p. 202-220, 2015. 
Tabela 2 - Tempos de atendimento (segundos) dos clientes nos caixas rápidos.

\begin{tabular}{|l|l|l|l|l|l|l|l|l|l|}
\hline 67 & 99 & 44 & 29 & 48 & 96 & 70 & 58 & 56 & 80 \\
\hline 95 & 38 & 47 & 108 & 54 & 122 & 42 & 50 & 93 & 117 \\
\hline 25 & 58 & 28 & 22 & 63 & 29 & 35 & 29 & 130 & 33 \\
\hline 20 & 127 & 45 & 51 & 108 & 88 & 59 & 41 & 21 & 58 \\
\hline 105 & 84 & 40 & 101 & 83 & 53 & 35 & 92 & 28 & 56 \\
\hline 32 & 34 & 87 & 124 & 52 & 176 & 57 & 368 & 66 & 70 \\
\hline 74 & 31 & 40 & 189 & 46 & 28 & 61 & 40 & 77 & 74 \\
\hline 33 & 52 & 67 & 86 & 40 & 46 & 27 & 54 & 126 & 76 \\
\hline 73 & 50 & 82 & 89 & 41 & 24 & 126 & 99 & 21 & 15 \\
\hline 45 & 36 & 45 & 57 & 32 & 60 & 210 & 44 & 55 & 65 \\
\hline 81 & 86 & 55 & 50 & 39 & 165 & 32 & 161 & 76 & 67 \\
\hline 61 & 34 & 40 & 73 & 42 & 73 & 60 & 132 & 61 & 52 \\
\hline 110 & 46 & 59 & 71 & 100 & 74 & 39 & 38 & 111 & 44 \\
\hline 35 & 79 & 182 & 76 & 65 & 94 & 59 & 56 & 79 & 8 \\
\hline 46 & 76 & 45 & 45 & 55 & 60 & 106 & 53 & 42 & 85 \\
\hline
\end{tabular}

Os resultados dos ajustes das distribuições de probabilidade fornecidos pelo Input Analyzer confirmam que as distribuições de probabilidade Exponencial aderem aos dados referentes aos entre chegadas, no entanto a Distribuição Normal não se apresentou adequada para os dados referentes aos tempos de atendimento, sendo neste caso utilizada a opção Fit All do Input Analyzer para identificar a distribuição que melhor adere aos dados coletados, conforme por ser visualizado no Quadro 2.

ReLAInEP - Revista Latino-America de Inovação e Engenharia de Produção, Curitiba, PR, Brasil, v. 3, n. 4, p. 202-220, 2015. 
Quadro 2 - Ajuste das distribuições de probabilidade.

\begin{tabular}{|c|c|c|}
\hline Tempos entre Chegadas & \multicolumn{2}{|l|}{ Tempos de Atendimento } \\
\hline \multirow{3}{*}{$\begin{array}{l}\text { Distribution: Exponential } \\
\text { Expression:-0.001+EXPO(43.4) } \\
\text { Square Error: } 0.002565\end{array}$} & Distribution: Normal & Distribution: Gamma \\
\hline & Expression: NORM $(66.2,35.9)$ & Expression:8+GAMM $(25.9,2.25)$ \\
\hline & Square Error: 0.020862 & Square Error: 0.005908 \\
\hline Chi Square Test & Chi S & Chi Square Test \\
\hline Number of intervals $=6$ & Number of intervals $=7$ & Number of intervals $=7$ \\
\hline Degrees of freedom $=4$ & Degrees of freedom $=4$ & Degrees of freedom $=4$ \\
\hline Test Statistic $=2.96$ & Test Statistic $=23.9$ & Test Statistic $=6.71$ \\
\hline Corresponding $p$-value $=0.569$ & $\begin{array}{c}\text { Corresponding } \\
<0.005\end{array}$ & Corresponding p-value \\
\hline \multirow{2}{*}{$\begin{array}{l}\text { Kolmogorov-Smirnov Test } \\
\text { Test Statistic }=0.0384\end{array}$} & & Kolmogorov-Smirnov Test \\
\hline & Kolmogorov-Smirnov Test & Test Statistic $=0.0719$ \\
\hline Corresponding p-value $>0.15$ & Test Statistic $=0.17$ & \multirow[t]{2}{*}{ Corresponding p-value } \\
\hline \multicolumn{2}{|l|}{$\begin{array}{l}\text { Data Summary: } \\
\text { Number of Data Points } \quad=150\end{array}$} & \\
\hline Number of Data Points $\quad=150$ & \multirow[b]{2}{*}{ Data Summary: } & \multirow{2}{*}{$\begin{array}{l}\text { Number of Data Points }=150 \\
\text { Min Data Value }=8\end{array}$} \\
\hline Min Data Value $=0$ & & \\
\hline Max Data Value $=206$ & Number of Data Points & Max Data Value $=210$ \\
\hline $\begin{array}{l}\text { Sample Mean }=43.4 \\
\text { Sample Std Dev }=42.1\end{array}$ & Min Data Value $=8$ & Sample Mean = 66.2 \\
\hline Sample Std Dev $=42.1$ & $\begin{array}{l}\text { Max Data Value }=210 \\
\text { Sample Mean }=66.2\end{array}$ & Sample Std Dev = 36 \\
\hline Histogram Summary & Sample Std Dev $=36$ & Histogram Summary \\
\hline Histogram Range $=-0.001$ to 206 & Histooram Summary & Histogram Range $=8$ to 210 \\
\hline & $\begin{array}{l}\text { Histogram Range }=8 \text { to } 210 \\
\text { Number of Intervals }=12\end{array}$ & \\
\hline
\end{tabular}

Fonte: input analyzer

Diante do exposto, tomamos para a modelagem do sistema de filas dos caixas rápidos, os seguintes parâmetros: i) Tempos entre Chegadas: -0.001 + EXPO (43.4) segundos; e ii) Tempos de Atendimentos: 8 + GAMM $(25.9,2.25)$ segundos.

\subsection{Modelagem e Simulação Computacional do Sistema de Filas}

Como o sistema de filas estudado, consiste em clientes entrando na fila, aguardando caso os caixas estejam ocupados, sendo então atendidos e deixando o sistema, para a modelagem do mesmo no Software Arena foram utilizados os módulos de fluxograma Create, Process e Dispose, conforme ilustrado na Figura 4

ReLAInEP - Revista Latino-America de Inovação e Engenharia de Produção, Curitiba, PR, Brasil, v. 3, n. 4, p. 202-220, 2015. 


\section{ReLAInEP}

Figura 4 - Templates utilizados na modelagem do sistema de filas

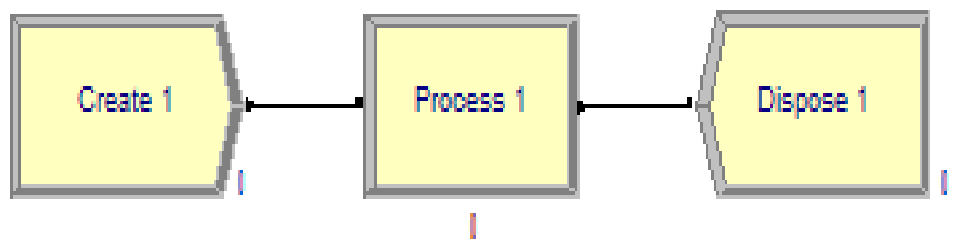

Fonte: Arena 14.0

De modo a representar o sistema em estudo, o módulo Create foi denominado Chegada de Clientes, o módulo Process foi denominado Atendimento de Clientes e o módulo Dispose foi denominado Saída de Clientes, conforme ilustrado na Figura 5.

Figura 5 - Sistema de filas dos caixas rápidos do supermercado x

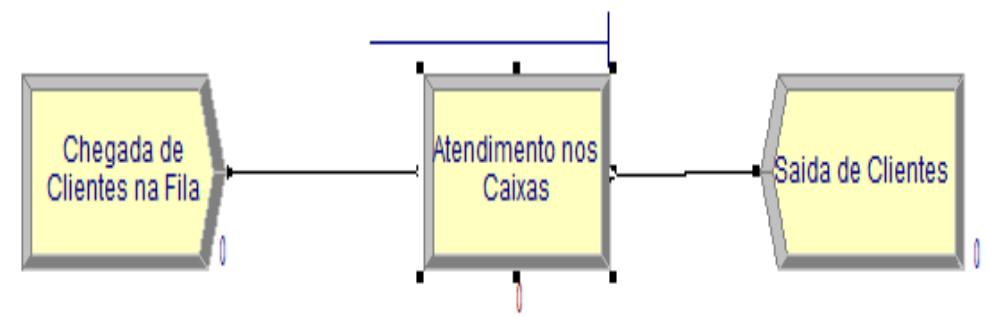

Fonte: Arena 14.0

Definidos e nomeados os módulos necessários para a representação do sistema no Software Arena, partiu-se para a configuração dos mesmos, conforme segue:

Na configuração do módulo Create foram considerados os seguintes parâmetros: i) Entity Type: Cliente; ii) Time Between Arrivals: Expression -0.001 + EXPO(43.4) segundos; iii) Entities per Arrival: 1; iv) Maximal Arrivals: infinite; e v) First Creation: 0,0. A Figura 6 ilustra a configuração do Módulo Create.

ReLAInEP - Revista Latino-America de Inovação e Engenharia de Produção, Curitiba, PR, Brasil, v. 3, n. 4, p. 202-220, 2015. 


\section{ReLAInEF}

Figura 6 - configuração do módulo create

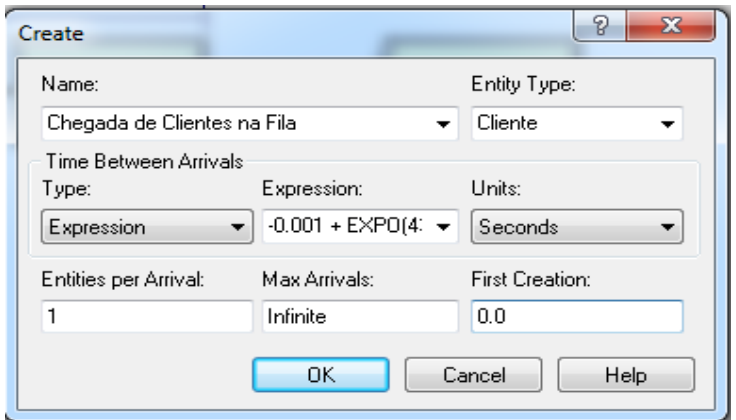

Fonte: Arena 14.0

Na configuração do módulo Process, nomeado Atendimento de Clientes, foram considerados os seguintes parâmetros: i) Type: Standard; ii) Action: Seize-Delay-Release; iii) Priority: Medium; iv) Resources: Caixa e 2 Operador de Caixa; v) Delay Type: 8 + GAMM (25.9, 2.25) segundos. A Figura 7 a seguir ilustra a configuração do Módulo Process. Foi utilizado o módulo de dados Resource para informar o número de caixas atendendo no período simulado. A configuração do módulo Resouce por ser visualizada na Figura 8.

Figura 7 - Configuração do módulo process

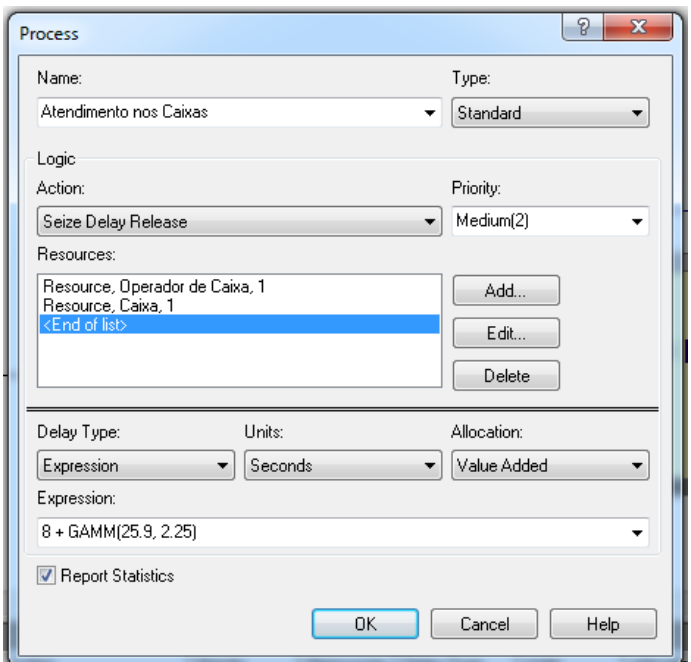

Fonte: Arena 14.0

ReLAInEP - Revista Latino-America de Inovação e Engenharia de Produção, Curitiba, PR, Brasil, v. 3, n. 4, p. 202-220, 2015. 


\section{ReLAlnEP}

Figura 8 - Configuração do módulo resource

\begin{tabular}{|c|c|c|c|c|c|c|c|c|c|}
\hline \multicolumn{10}{|c|}{ Resource - Basic Process } \\
\hline & Name & Type & Capacity & Busy / Hour & Idle / Hour & Per Use & StateSet Name & Failures & Report Statistics \\
\hline 1 & Caixa & Fixed Capacity & 2 & 0.0 & 0.0 & 0.0 & & 0 rows & $\sqrt{v}$ \\
\hline 2 & Operador de Caixa & Fixed Capacity & 2 & 0.0 & 0.0 & 0.0 & & 0 rows & $\sqrt{v}$ \\
\hline
\end{tabular}

Fonte: Arena 14.0

Por último adiciona-se o bloco Dispose que é apenas renomeado como Saída dos Clientes, conforme Figura 9

Figura 9 - Configuração do módulo dispose

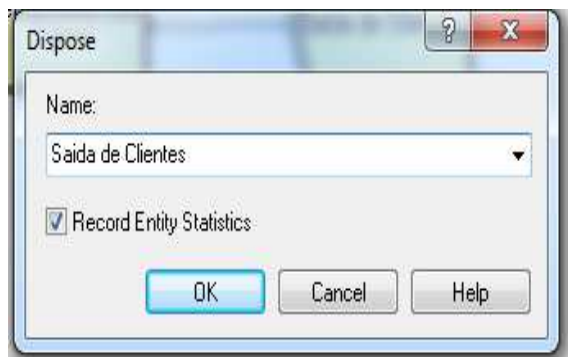

Fonte: Arena 14.0

As configurações gerais da simulação, para um período 3600 segundos podem ser visualizados na Figura 10.

Figura 10 - Configurações gerais da simulação

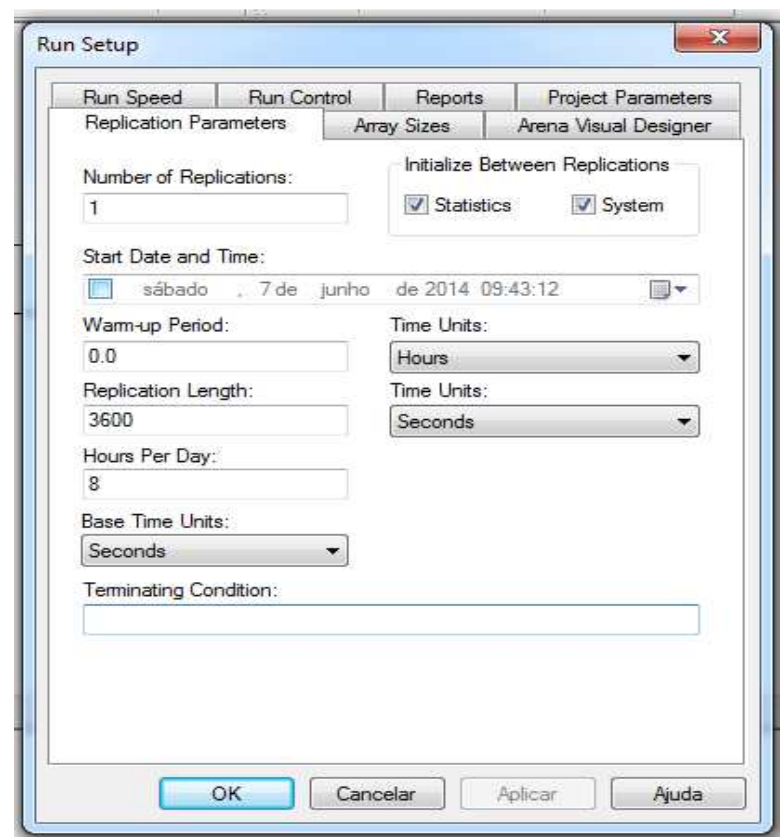

ReLAInEP - Revista Latino-America de Inovação e Engenharia de Produção, Curitiba, PR, Brasil, v. 3, n. 4, p. 202-220, 2015. 


\section{ReLAlnEP}

\subsection{Resultados e Discussões da Simulação do Sistema de Filas}

O Supermercado $\mathrm{X}$ conta com 4 caixas rápidos, mas geralmente opera com 2 caixas. No entanto, para avaliar o comportamento do sistema de filas dos caixas rápidos, foi executada 1 replicação da simulação do sistema em estudo operando durante um período de 1 hora (3600 segundos), para as seguintes configurações: i) 2 caixas rápidos; ii) 3 caixas rápidos; e iii) 4 caixas rápidos.

$\mathrm{Na}$ simulação do sistema de filas com 2 caixas rápidos operando em um período de 3600 segundos, entraram em fila 78 clientes e deixaram o sistema 76 clientes, sendo verificada uma taxa de ocupação dos caixas igual a 80,92\%. Os demais resultados da simulação para este cenário são apresentados no Quadro 3.

Quadro 3 - Resultados da simulação para operação de 2 caixas rápidos

\begin{tabular}{|llll|}
\hline Medidas de Efetividade (Unidade de Medida) & Média & Mínimo & Máximo \\
\hline Tempo na Fila (Segundos) & 64,42 & 0,00 & 266,83 \\
Tempo no Atendimento (Segundos) & 72,12 & 15,63 & 231,33 \\
Tempo no Sistema (Segundos) & 140,56 & 21,16 & 393,20 \\
Tamanho da Fila (Número de Clientes) & 1,36 & 0,00 & 8,00 \\
\hline
\end{tabular}

$\mathrm{Na}$ simulação do sistema de filas com 3 caixas rápidos operando em um período de 3600 segundos, entraram em fila 72 clientes e deixaram o sistema 71 clientes, sendo verificada uma taxa de ocupação dos caixas igual a 48,90\%. Os demais resultados da simulação para este cenário são apresentados no Quadro 4.

Quadro 4 - Resultados da simulação para operação de 3 caixas rápidos

\begin{tabular}{|llll|}
\hline Medidas de Efetividade (Unidade de Medida) & Média & Mínimo & Máximo \\
\hline Tempo na Fila (Segundos) & 5,01 & 0,00 & 59,14 \\
Tempo no Atendimento (Segundos) & 72,99 & 13,84 & 207,56 \\
Tempo no Sistema (Segundos) & 78,08 & 15,63 & 207,5 \\
Tamanho da Fila (Número de Clientes) & 0,10 & 0,00 & 2,00 \\
\hline
\end{tabular}

$\mathrm{Na}$ simulação do sistema de filas com 4 caixas rápidos operando em um período de 3600 segundos, entraram em fila 72 clientes e deixaram o sistema 71 clientes, sendo verificada uma taxa de ocupação dos caixas igual a 36,89\%. Os demais resultados da simulação para este cenário são apresentados no Quadro 5.

ReLAInEP - Revista Latino-America de Inovação e Engenharia de Produção, Curitiba, PR, Brasil, v. 3, n. 4, p. 202-220, 2015. 
Quadro 5 - Resultados da simulação para operação de 5 caixas rápidos

\begin{tabular}{|llll|}
\hline Medidas de Efetividade (Unidade de Medida) & Média & Mínimo & Máximo \\
\hline Tempo na Fila (Segundos) & 0,79 & 0,00 & 21,13 \\
Tempo no Atendimento (Segundos) & 73,38 & 13,84 & 207,56 \\
Tempo no Sistema (Segundos) & 74,17 & 15,63 & 207,56 \\
Tamanho da Fila (Número de Clientes) & 0,015 & 0,00 & 2,00 \\
\hline
\end{tabular}

Com base nos resultados da simulação apresentada no Quadro 3 podemos verificar que existem períodos na simulação em que não ocorre formação de filas e outros períodos em que o tempo que um cliente permanece em fila quase 5 vezes o tempo de que leva seu atendimento no caixa. Por se tratar de um estudo probabilístico, estes valores podem sofrer alterações à medida que novas rodadas (replicações) de simulações sejam executadas. No entanto, o número médio de clientes em clientes em fila pode ser considerado pequeno, quando comparado com o número de clientes que chegam ao estabelecimento em uma hora.

Os resultados do Quadro 4 mostram uma redução nas medidas de desempenho do sistema, quando comparados o cenário com 2 e 3 caixas rápidos em operação. Verificamos uma redução de 32,02\% na taxa de ocupação dos caixas. Verificamos também que houve uma redução de $92,22 \%$ no tempo médio dos clientes em fila, $94,44 \%$ no tempo médio dos clientes no sistema e 92,64\% do número médio de clientes na fila. Este cenário pode ser adotado em momentos em que a afluência de clientes ao Supermercado $\mathrm{X}$ torna-se maior, como por exemplo, vésperas de feriados.

Os resultados do Quadro 5 mostram uma redução nas medidas de desempenho do sistema, quando comparados o cenário com 3 e 4 caixas rápidos em operação. Verificamos uma redução de 32,02\% na taxa de ocupação dos caixas. Verificamos também que houve uma redução de $84,23 \%$ no tempo médio dos clientes em fila, 5,00\% no tempo médio dos clientes no sistema e $85,00 \%$ do número médio de clientes na fila. No entanto, o cenário com 4 caixas rápidos pode não ser o mais adequado para o

Supermercado X, devendo, portanto, ser efetuada uma análise dos custos relacionados a operação do sistema com 4 caixas, uma vez que deve haver um equilíbrio entre a satisfação dos clientes e os custos pagos para obter tal satisfação.

ReLAInEP - Revista Latino-America de Inovação e Engenharia de Produção, Curitiba, PR, Brasil, v. 3, n. 4, p. 202-220, 2015. 


\section{CONSIDERAÇÕES FINAIS}

Atualmente esperar em filas é muito comum, seja para realizar compras no supermercado, pagar uma conta no banco, esperar uma consulta nos hospitais entre outros. O estudo das filas trata dos problemas de congestionamento de sistemas, onde a característica principal é a presença de clientes em busca de serviços.

O Supermercado $\mathrm{X}$ tem ao todo 4 caixas rápidos, mas em geral opera com apenas 2. Se o Supermercado X operar com 3 caixas, ao invés de 2 caixas, o número médio de clientes na fila passará de 1,36 para 0,10, o tempo médio dos clientes na fila passará de 64,42 segundos para 5,01 segundos e o tempo médio dos clientes no sistema passará de 140,56 segundos para 78,08 segundos.

Operar com 4 caixas, pode não ser economicamente viável para o Supermercado X, portanto, sugere-se para estudos futuros, que sejam efetuadas as análises dos custos de operação do sistema de filas com 2, 3 e 4 caixas.

O sistema de atendimento dos caixas rápidos apresenta fila única, sendo que foi verificada nas observações in loco a existência de um pilar que faz com que a fila fique posicionada a esquerda dos caixas, fazendo com que muitos clientes fiquem esperando para serem atendidos pelo Caixa 1 pois não veem o Caixa 2 desocupado, o que provoca atraso no tempo de atendimento. Sugere-se para esta disfunção, reposicionar os caixas, para que o pilar fique entre os caixas, e que sejam formadas filas individuais para cada caixa em operação. Depois de efetuada estas alterações, sugere-se que sejam efetuadas novas coletas de dados para realizar simulação com este novo cenário.

Uma vez que nos caixas rápidos o operador do caixa é responsável por embalar as mercadorias, sugere-se que em períodos de maior afluência de clientes no Supermercado X, seja colocado um colaborador extra para empacotar as mercadorias, de modo que não ocorram aumentos consideráveis nos tempos de atendimento dos caixas.

Os autores deste trabalho externam a sua gratidão a todos os assessores do Sistema Líder A, pelo trabalho primoroso no âmbito da sustentabilidade na construção. Agradecemos também o apoio financeiro do Programa de Educação Tutorial - PET e do programa Ciência sem Fronteiras, ambos do Governo Federal, com a concessão de bolsas de estudo.

ReLAInEP - Revista Latino-America de Inovação e Engenharia de Produção, Curitiba, PR, Brasil, v. 3, n. 4, p. 202-220, 2015. 


\section{REFERÊNCIAS}

ABEPRO. Associação Brasileira de Engenharia de Produção. Áreas e Subáreas da Engenharia de Produção. 2008. Disponível

em:<http://www.abepro.org.br/interna.asp?p=399\&m=424\&ss=1\&c=362>. Acesso em 07 Jun. 2014.

ALENCAR, E. D. M., et al. Modelagem matemática fundamentada em teoria das filas: um estudo do desempenho do sistema de filas em caixas rápidos de supermercado. In: Simpósio de Engenharia de Produção (XVII SIMPEP), 17, 2010, Bauru - SP. Anais... Bauru - SP, 2010.

ANDRADE, E, L. Introdução à Pesquisa Operacional: Métodos para Análise de Decisão. Rio de Janeiro: LTC - Livros Técnicos e Científicos, 1989.

ANDRADE, E. L. Introdução à Pesquisa Operacional: métodos e modelos para a análise de decisão. Rio de Janeiro, RJ. $2^{\circ}$ Edição: 1998.

ANDRADE, E. L. Introdução à Pesquisa Operacional: métodos e modelos para a análise de decisão. Rio de Janeiro, RJ. $2^{\circ}$ Edição: 2000.

ANDRADE, E.L.de. Introdução à pesquisa operacional: métodos e modelos para a análise de decisões. $3^{\text {a }}$ Edição. Rio de Janeiro: LTC - Livros técnicos e Científicos, 2002.

ARENALES, M. et al. Pesquisa Operacional. 526 p. Rio de Janeiro: Elsevier: ABEPRO, 2011.

ASCARP, L. P. C.; TORRES, A. A. V. B., SILVA, A. L. Simulação como ferramenta de tomada de decisão do processo de atendimento em empresa de varejo. Revista Race. Unoesc, v. 12, n.2, p. 395-430, jul./dez. 2013.

ASSIS, R. Anexo VI “Técnicas Básicas de Simulação” do livro “Apoio à Decisão em Manutenção na Gestão de Activos Físicos”. LIDEL, 2010. Disponível

em:<http://www.rassis.com/artigos/Resumo\%20Simulacao.pdf $>$. Acesso em: 08 Junho de 2014.

BRIGHENTI, J. R. N. Simulação e Otimização de uma Linha de Manufatura em Fase de Projeto. (Dissertação) Universidade Federal de Itajubá. Programa de Pós-Graduação em Engenharia de Produção. Itajubá, 2006. Disponível

em:<http://www.iepg.unifei.edu.br/arnaldo/download/dissertacoes/Renato\%20Brighenti.pdf >. Acesso em: 08 Junho de 2014.

BRONSON, Richard. Pesquisa Operacional. São Paulo: McGraw-Hill do Brasil, 1985.

ELLENRIEDER, Alberto Von. Pesquisa Operacional. Rio de Janeiro: Almeida Neves, 1971.

FOGLIATTI, M. C.; MATTOS, N. M. C. Teoria de Filas. Rio de Janeiro: Interciência, 2007.

GAN, S. Como funcionam as filas?. Super Interessante, editora Abril: Dezembro, 2004. Disponível em: < http://super.abril.com.br/ciencia/como-funcionam-filas445066.shtml>. Acesso em: 24 out. 2013.

ReLAInEP - Revista Latino-America de Inovação e Engenharia de Produção, Curitiba, PR, Brasil, v. 3, n. 4, p. 202-220, 2015. 
HILLIER, F. S.; LIEBERMAN, G. J. Introdução à pesquisa operacional. $8^{\mathrm{a}}$ Edição. Porto Alegre: AMGH, 2010.

MIYAGI, P. E. Introdução a Simulação Discreta. Escola Politécnica da Universidade de São Paulo. Departamento de Engenharia Mecatrônica e de Sistemas Mecânicos. São Paulo, 2006. Disponível em: <http://minerva.ufpel.edu.br/ alejandro.martins/dis/2012_2/simulacao/Apostila_Simulaca o.pdf $>$. Acesso em: 08 Junho de 2014.

MORAIS, M.F. Modelagem e Simulação de sistemas. Campo Mourão, Universidade Estadual do Paraná (Unespar) - Campus de Campo Mourão, DEP, 2013. 20 p. Apostila.

MOREIRA, D. A. Pesquisa Operacional: Curso introdutório. São Paulo: Thomson Learning, 2007.

PEREIRA JUNIOR, W. M. Teoria das Filas e Simulações. Ituiutaba: Universidade do Estado de Minas Gerais, 2010. Apostila.

PRADO, D. Usando o ARENA em simulação. $3^{\text {a }}$ Edição. Belo Horizonte: Editora de desenvolvimento gerencial, 1999.

RINALDI, J. G. S. A importância da rapidez de atendimento de caixas de supermercado: um estudo de caso utilizando um modelo analítico de filas com trocas. Tese de Doutorado (Doutorado em Engenharia de Produção) - Programa de Pós Graduação em Engenharia de Produção, Universidade Federal de São Carlos, São Carlos-SP, 2007.

SCHNEIDERS, C. F. DESCONZI, A., REPPENTHAL, I. L. Estudo das filas em um supermercado.In: SALÃO DE PESQUISA SETREM (X SETREM), 10, 2012, Três de Maio - RS. Anais... Três de Maio - RS, 2012.

SILVEIRA, A. P.; SILVA, C. R.; BELARMINO, D. G. O Software ARENA. Centro Universitário do Sul de Minas - UNIS-MG. Bacharelado em Ciência da Computação. Varginha, 2006. Disponível em:<http://www.cienciadacomputacao.unis.edu.br/files/2010/10/013_Arena.pdf>. Acesso em: 08 Junho de 2014.

SLACK, N.; CHAMBER, S.; HARDLAND, C.; HARRISON, A. e JOHNSTON, R. Administração da Produção. São Paulo: Atlas, 1999.

TAHA, H. A. Pesquisa Operacional: uma visão geral. $8^{\circ}$ Edição. São Paulo: Pearson Prentice Hall, 2007.

ReLAInEP - Revista Latino-America de Inovação e Engenharia de Produção, Curitiba, PR, Brasil, v. 3, n. 4, p. 202-220, 2015. 


\section{ReLAInEP}

ReLAInEP - Revista Latino-America de Inovação e Engenharia de Produção, Curitiba, PR, Brasil, v. 3, n. 4, p. 202-220, 2015. 